\title{
Nonlinear Characteristic of Spherical Joints with Clearance
}

\author{
Zhang Jing ${ }^{1}$, Guo Hong-Wei², Liu Rong-Qiang², Deng Zong-Quan²
}

\begin{abstract}
Joints in deployable structures can degrade the stiffness and the stability of spacecraft. In this study, the nonlinear stiffness of spherical joints is investigated. The traditional contact model of spherical joints based on non-conforming contact assumption is presented. A new contact model for spherical joints based on the Winkler model and geometric constraints is established to calculate the stiffness of spherical joints with small clearances. The finite element model (FEM) of spherical joints is built to evaluate the accuracy of the theoretical model. The effects of the clearance and the contact force of spherical joints on the deformation of joints are investigated. When compared with FEM results, the error of the new spherical model is smaller than that of the traditional contact model when the clearance of spherical joint is not excessively large. The new contact model for spherical joint is more accurate than the traditional contact model when the clearance of spherical joint is large.
\end{abstract}

KEYWORDS: Deployable structure, Nonlinear characteristic, Joint, Stiffness.

\section{INTRODUCTION}

Given the need of sending large payloads and high volume of launchers in space missions, deployable structures as supporting ones have been widely used in spacecraft (Puig et al., 2010; Gruber et al., 2007). The deployable mast for solar sails has reached the range of $100-1,000 \mathrm{~m}$ (Brown, 2011). The large deployable structures usually contain a large number of joints to achieve the smooth rotation of the structure parts. Joint clearance often generates nonlinear characteristics, such as impact or contact, which affects the stability and the accuracy of deployable structures (Qi et al., 2010).

The dynamic performance of deployable structures is important for space structure design. In the first type of dynamic analysis, joint clearance is neglected. Shin and Lee (2000) proposed an improved approach to update the initial finite element model (FEM) for space structures. The second type of analysis considers the impact and free-play generated by the joint clearance. Sarkar modeled the space trusses with nonlinear force caused by joint that is expressed by harmonic function (Sarkar et al., 2004). (Li et al., 2011) investigated the transient dynamic response of plane mechanics and analyzed the effect of clearance on the dynamic performance of structures by utilizing gap element to model clearance joint in ANSYS software. Joint clearance is considered not only in dynamic analysis of space trusses, but also in mechanism analysis. Bauchau and Rodriguez (2002) considered three-dimensional configuration of spherical joint and studied the effect of clearance, lubrication and friction phenomena on the dynamic response of flexible multibody systems.

The contact model of a joint is mainly based on elastic theory and finite element theory. Hertz law is proposed to model the contact of joints (Johnson, 1985). Lankarani and Nikravesh (1990) presented a modified Hertzian-based contact

\footnotetext{
三 1.Taiyuan University of Technology - College of Mechanical Engineering - Taiyuan - China 2.Harbin Institute of Technology - School of Mechatronics Engineering Harbin - China.

Author for correspondence: Zhang Jing | Taiyuan University of Technology - College of Mechanical Engineering | 79 - Yingze West Street - Wanbolin District | Zip Code: 030024 - Taiyuan - China | Email: free1985216@163.com

Received: 01/16/2015 | Accepted: 03/26/2015
} 
law, which includes a damping term to account for the energy dissipation. Tian et al. (2009) calculated the contact force of spherical joint in spatial flexible multibody systems by using the continuous contact model proposed by Lankarani and Nikravesh. Boltachev et al. (2012) studied the elastic interaction of spherical particles whose stress distribution is determined by a rod model, which was applied in a more wide range of deformation calculation when compared with the classical Hertz law. Liu et al. (2006) built an approximate contact model of spherical joints with clearance that is based on distributed elastic forces. Fang et al. (2015) proposed an accurate theoretical solution to calculate the conformal contact pressure distribution in spherical plain bearings through the equivalent force principle. Bai and Zhao (2013) presented a new force model of the revolute joint with clearance for planar mechanical systems, which is a hybrid model of the Lankarani-Nikravesh and the improved elastic foundation models. Rajaei and Ahmadian (2014) used the Iwan model to simulate stick-micro/macro slip behavior involved in a friction contact. Pereira et al. (2014) estimated the applicability of the cylindrical contact models proposed in previous studies. The research on contact model is mainly based on the Hertz theory. Although energy dissipation, slip, and friction in the contact solids are considered, the accurate of the contact models usually used in mechanism simulation is not completely studied. The models based on Hertz model are not suitable for the conforming contact of joint because Hertz model is based on non-conforming assumption.

Finite element method is the other method to investigate the contact of joints. Cannon and Howell (2005) used pseudorigid-body model to predict the behavior of the conforming contact-aided revolute joint and validated the model by finite element analysis. Liu et al. (2007) established an approximate model for the contact problem of cylindrical joints by introducing appropriate assumptions and by analyzing the FEM numerical results. Because the elastic contact calculation by implementing finite element software depends on the user's choice in some parameters such as normal contact stiffness and penetration limit, Hattori and Serpa (2015) predicted the values of some parameters by investigating the artificial neural networks. Big-Alabo et al. (2015) conducted elastoplastic analysis for a spherical impactor. Finite element method can be used to analyze various contact conditions. However, the major limitation of this type of analysis is time-consuming. This method can hardly be combined with the simulation of mechanism. Thus, an accurate conforming model for a joint is important to mechanism simulation.

In this paper, the classic contact model for spherical joint is presented. The following four assumptions are used as basis: (i) the surfaces are continuous and non-conforming; (ii) the strains are small; (iii) each solid can be considered as an elastic half-space; (iv) the surfaces are frictionless. The non-conforming in the assumptions means that the contact width is significantly smaller than the equivalent radius. The non-conforming assumption for Hertz model is not suitable for the spherical joint with small clearance, because, in general, the contact width of the joint is not significantly smaller than equivalent radius. Therefore, the classic contact model cannot be adequately used to calculate the stiffness of spherical joints in deployable structures, because the clearance is usually small for satisfying the high accuracy of the supporting structures. Based on the geometric constraint model of revolute joint, the geometric model for spherical joint is given. The contact model for spherical joints is built based on Winkler model. The FEM for spherical joint is established to evaluate the accuracy of the proposed contact model.

\section{CONTACT MODEL FOR SPHERICAL JOINTS \\ CLASSICAL CONTACT MODEL}

The contact area of rotation is a circle. The four assumptions cited before exist in the classical contact model. When a normal load $P$ is applied, two spherical solids are shown in the cross section after deformation in Fig. $1 . R_{1}$ and $R_{2}$ are the radius of the two spheres; $u_{z}(r)$ is the total deformation of the two spheres on the contact circle of radius $r$. The total deformation of the two contact solids is $\delta=\delta_{1}+\delta_{2}=u_{z}(0)$, where $\delta_{1}$ and $\delta_{2}$ are the deformation of the $\mathrm{N}^{\text {o. }} 1$ solid and the $\mathrm{N}^{\mathrm{o}} \cdot 2$ solid on the centre of the contact area.

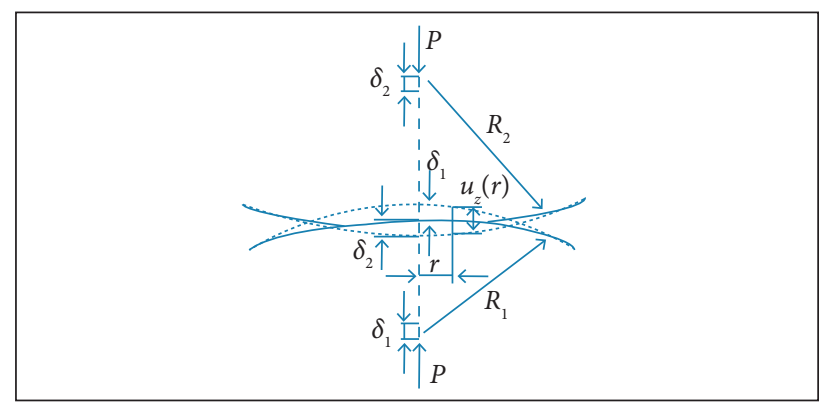

Figure 1. Hertz model for two spherical solids. 
The total deformation of the two spheres on the contact circle of radius $r$ can be expressed as:

$u_{z}(r)=\delta-\frac{1}{2 R} r^{2}$

where:

$R$ : equivalent radius; $1 / R=1 / R_{1}+1 / R_{2}$ when the two solids are convex.

The Hertz contact force of spherical joint satisfies:

$$
p(r)=p_{0} \sqrt{1-\left(\frac{r}{a}\right)^{2}}
$$

where:

$a$ : the contact width at the center of contact area; $p_{0}$ : contact pressure at the center of contact area.

The relationship between the contact deflection of the sphere and the contact force is presented as (Johnson, 1985):

$$
\delta=\left(\frac{9 P^{2}}{16 R E^{2}}\right)^{\frac{1}{3}}
$$

where:

$E$ : composite modulus $1 / E=\left(1-v_{1}^{2}\right) / E_{1}+\left(1-v_{2}{ }^{2}\right) / E_{2} ; E_{1}$ and $E_{2}$ : the elastic modulus of the two contact solids; $v_{1}$ and $v_{1}$ : the Poisson ratios of the two contact solids.

Based on the simplification of Eq. 3, the contact force can be given by:

$$
P=\frac{4 E \delta}{3} \sqrt{\delta R}
$$

\section{CONTACT MODEL OF SPHERICAL JOINT BASED ON THE WINKLER MODEL AND GEOMETRIC CONSTRAINTS}

The Hertz contact model is rarely used to obtain highaccuracy contact stiffness of the spherical joint with small clearance because the non-conforming contact is assumed in Hertz contact model of sphere. The spherical joint and the contact model of this joint are shown in Fig. 2; $r$ can vary from 0 to $a$.

The elastic half-space can be replaced with Winkler elastic base. The simplified model is shown in Fig. 3. The contact forces of the points on the contact circle of radius $r$ can be expressed as (Johnson, 1985):
$p(r)=\frac{E}{h} u_{z}(r)$

where:

$h$ : thickness of the spherical shell.

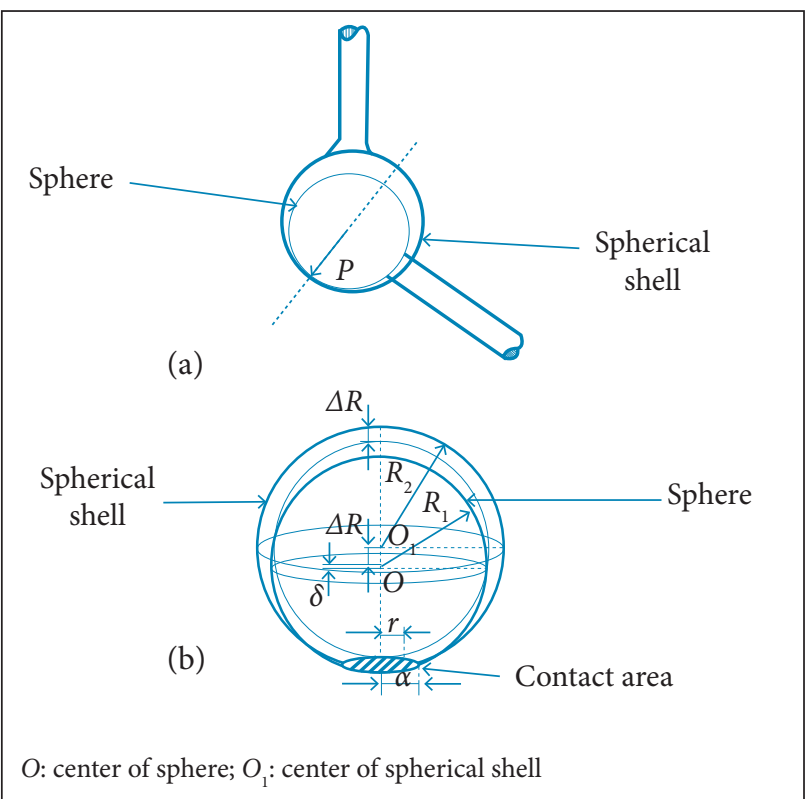

Figure 2. Contact of spherical joint. (a) Spherical joint; (b) Contact model for spherical joint.

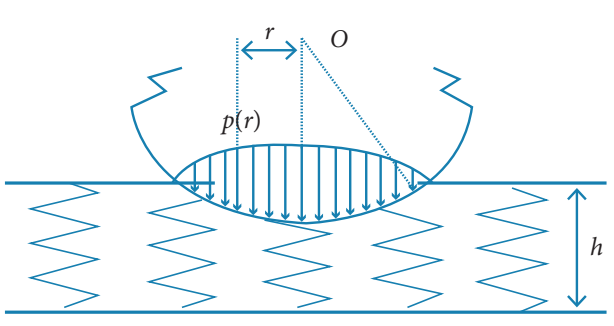

Figure 3. Simplified contact model for spherical joints with clearance.

The contact force of the central point of the contact area can be expressed as:

$$
p_{0}=\frac{E}{h} \delta
$$

The contact force given by traditional Winkler model is expressed as:

$$
P=\frac{\pi E \delta^{2} R}{h}
$$


The pressure distribution of the contact of revolution solids is presented in Hertz model, which is validated. Based on the distribution of the pressure given by Hertz, the total contact force can be expressed as:

$$
P=\int_{0}^{a} p(r) 2 \pi r \mathrm{~d} r=\frac{2}{3} p_{0} \pi a^{2}
$$

Based on Eqs. 6 and 8, the relationship among the contact force, contact width, and the contact deflection can be given as:

$$
P=\frac{2 \pi E \delta}{3 h} a^{2}
$$

The contact width and the contact deflection in any rotation plane plotted by dashed line in Fig. 2b satisfy the relationship between contact deflection and contact width of revolute joint described in Liu et al. (2013). Taking the contact width expression into Eq. 9, the relationship between contact force and contact deflection can be given by:

$$
P=k_{\mathrm{j}} \delta=\delta \frac{2 \pi E}{3 h}\left[R_{2}^{2}-\frac{1}{4}\left(\frac{R_{2}^{2}-R_{1}^{2}}{\delta+\Delta R}+\delta+\Delta R\right)^{2}\right]
$$

\section{VERIFICATION OF THE PROPOSED CONTACT MODEL}

The FEM of spherical joint is built to verify the established contact model of spherical joint. The FEM is shown in Fig. 4, which is established by ANSYS. The type of the element is SOLID 45. The contact models of spherical joints with different clearances are established, where $R_{2}=5 \mathrm{~mm}, E=2.06 \times 10^{5} \mathrm{Mpa}$ and $h=5 \mathrm{~mm}$.

The comparison between theoretical model and the FEM for spherical joint is given in Fig. 5, which plots the relationship

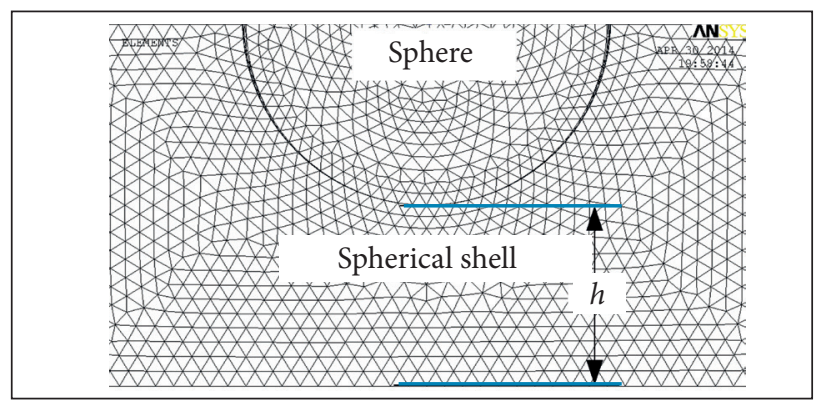

Figure 4. FEM for spherical joint.

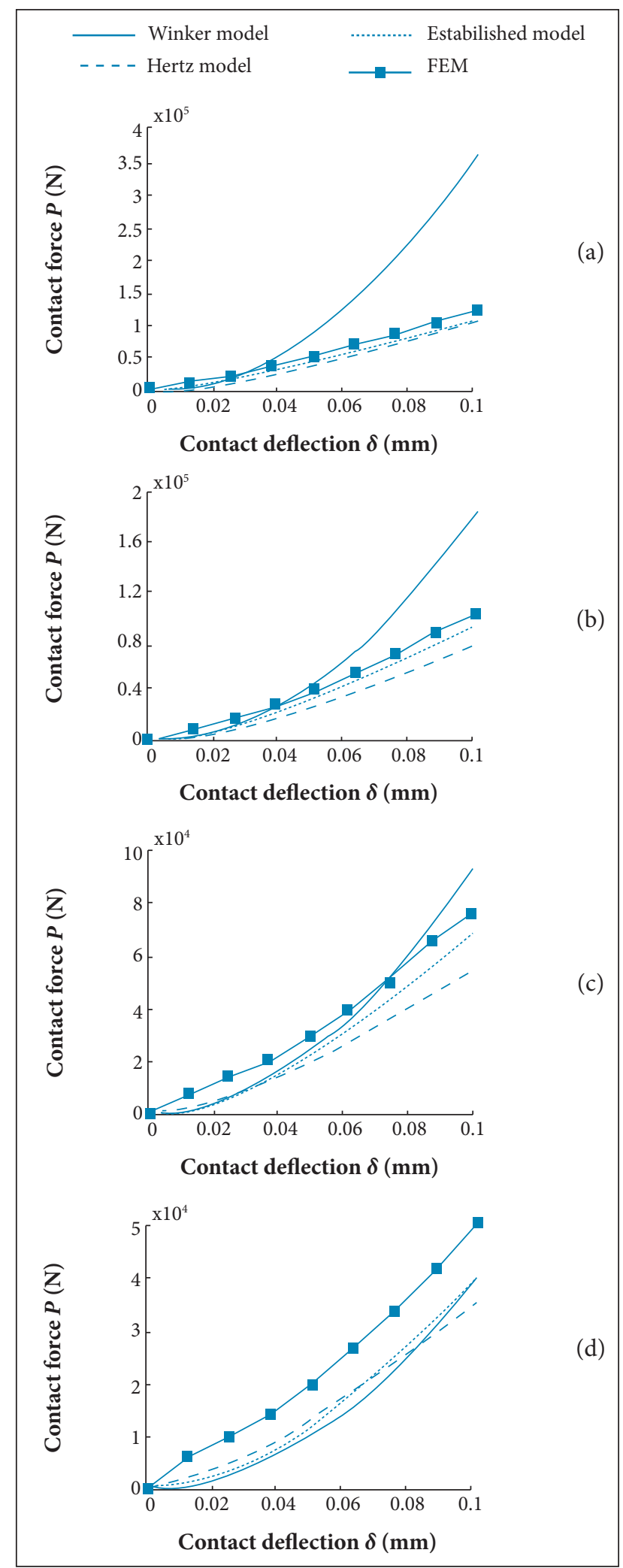

Figure 5. Comparison of the contact models for spherical joint. (a) $\Delta R=0.05 \mathrm{~mm}$; (b) $\Delta R=0.1 \mathrm{~mm}$; (c) $\Delta R=0.2 \mathrm{~mm}$; (d) $\Delta \mathrm{R}=0.5 \mathrm{~mm}$. 
between the contact force and the contact deflection of spherical joint. The stiffness of the joint decreases with the joint clearance. When the contact deflection is small, the error of the theory model is smaller than that of Hertz model. With the increase of the clearance of spherical joint, the error of the theory model in total contact deflection range is closed to that of the Hertz model. When the clearances of the spherical joints are $0.05 ; 0.1$; 0.2 and $0.5 \mathrm{~mm}$, respectively, the errors of the Hertz contact model compared with FEM are 94.1; 43.3; 29.0 and 49.5\%. The errors of the Hertz contact model compared with FEM are 30.5, 35.6, 38.6, and 40.4\%, respectively. Moreover, the errors of the theory model compared with FEM are 22.4, 23.1, 30.1, and 44.2\%, respectively. Thus, the established spherical model is accurate in terms of calculating the stiffness of the spherical joint contact.

\section{CONCLUSIONS}

Spherical joints are investigated because of their importance in the application of deployable structures in space missions. The nonlinear characteristic of spherical joints can affect the dynamic response of deployable structures. First, the traditional contact model of spherical joints is presented. The contact model is based on four assumptions. The model is suitable for joints with large clearance. Second, a new contact model for spherical joints is modeled based on the Winkler model and geometric constraints given in literature. Third, finite element models for spherical joint with different clearances are built. The simulation results are compared with the results of traditional model and those of the new model. The error of the new contact model is close to that of the traditional model when the clearance is small. The error of the new contact model is closed to that of the traditional model when the clearance is large. Therefore, the new model can be accurate in calculating the stiffness of spherical joints with clearance.

\section{ACKNOWLEDGEMENTS}

This research is financially supported by the Fundamental Research Funds for Central Universities (Grant No. HIT. NSRIF.2015050), Self-Planned Task (No. SKLRS201401A02) of State Key Laboratory of Robotics and System (HIT), Shanxi Provincial Higher School Outstanding Innovative Team (2011), The Qualified Personnel Foundation of Taiyuan University of Technology (No. tyut-rc201448a), and The Special/Youth Foundation of Taiyuan University of Technology (No. 2014TD040).

\section{REFERENCES}

Bai, Z.F. and Zhao, Y., 2013, "A Hybrid Contact Force Model of Revolute Joint with Clearance for Planar Mechanical Systems", International Journal of Non-linear Mechanics, Vol. 48, pp. 15-36. doi: 10.1016/j.ijnonlinmec.2012.07.003

Bauchau, O.A. and Rodriguez, J., 2002, "Modeling of Joints with Clearance in Flexible Multibody Systems", International Journal of Solids and Structures, Vol. 39, No. 1, pp. 41-63. doi: 10.1016/ S0020-7683(01)00186-X

Big-Alabo, A., Harrison, P. and Cartmell, M.P., 2015, "Contact Model for Elastoplastic Analysis of Half-Space Indentation by a Spherical Impactor", Computers \& Structures, Vol. 151, pp. 2029. doi: 10.1016/j.compstruc.2015.01.005

Boltachev, G.S., Volkov, N.B. and Zubarev, N.M., 2012, "Tangential Interaction of Elastic Spherical Particles in Contact", International Journal of Solids and Structures, Vol. 49, No. 15-16, pp. $2107-$ 2114. doi: 10.1016/j.ijsolstr.2012.04.013

Brown, M.A., 2011, "A Deployable Mast for Solar Sails in the Range of 100-1000 m", Advances in Space Research, Vol. 48, No. 11, pp. 1747-1753. doi: 10.1016/j.asr.2011.01.014

Cannon, J.R. and Howell, L.L., 2005, "A Compliant Contact-Aided Revolute Joint", Mechanism and Machine Theory, Vol. 40, No. 11, pp. 1273-1293. doi: 10.1016/j.mechmachtheory.2005.01.011
Fang, X., Zhang, C.H., Chen, X., Wang, Y.S. and Tan, Y.Y., 2015, "Newly Developed Theoretical Solution and Numerical Model for Conformal Contact Pressure Distribution and Free-Edge Effect in Spherical Plain Bearings", Tribology International, Vol. 84, pp. 4860. doi: 10.1016/j.triboint.2014.11.020

Gruber, P., Häuplik, S., Imhof, B., Özdemir, K., Waclavicek, R. and Perino, M.A., 2007, "Deployable Structures for a Human Lunar Base", Acta Astronautica, Vol. 61, No. 1-6, pp. 484-495. doi: 10.1016/j.actaastro.2007.01.055

Hattori, G. and Serpa, A.L., 2015, "Contact Stiffness Estimation in ANSYS Using Simplified Models and Artificial Neural Networks", Finite Elements in Analysis and Design, Vol. 97, pp. 43-53. doi: 10.1016/j.finel.2015.01.003

Johnson, K.L., 1985, “Contact Mechanics”, Cambridge University Press, Cambridge, UK.

Lankarani, H.M. and Nikravesh, P.E., 1990, "A Contact Force Model with Hysteresis Damping for Impact Analysis of Multibody Systems", Journal of Mechanical Design, Vol. 112, No. 3, pp. 369-376. doi: 10.1115/1.2912617

Li, T.J., Guo, J. and Cao, Y.Y., 2011, "Dynamic Characteristics Analysis of Deployable Space Structures Considering Joint Clearance", Acta Astronautica, Vol. 68, No. 7-8, pp. 974-983. doi: 10.1016/j.actaastro.2010.08.039 
Liu, C.S., Zhang, K. and Yang, L., 2006, "Normal Force-Displacement Relationship of Spherical Joints with Clearances", Journal of Computational and Nonlinear Dynamics, Vol. 1, No. 2, pp. 160-167. doi: $10.1115 / 1.2162872$

Liu, C.S., Zhang, K. and Yang, R., 2007, "The FEM Analysis and Approximate Model for Cylindrical Joints with Clearances", Mechanism and Machine Theory, Vol. 42, No. 2, pp. 183-197. doi: 10.1016/j. mechmachtheory.2006.02.006

Liu, R.Q., Zhang, J., Guo, H.W. and Deng, Z.Q., 2013, "Nonlinear Characteristics of Revolute Joints with Clearance", Journal of Aerospace Technology and Management, Vol. 5, No. 3, pp. 341-348. doi: 10.5028/ jatm.v5i3.236

Pereira, C., Ramalho, A. and Ambrosio, J., 2014, "Applicability Domain of Internal Cylindrical Contact Force Models", Mechanism and Machine Theory, Vol. 78, pp. 141-157. doi: 10.1016/j.mechmachtheory.2014.03.010

Puig, L., Barton, A. and Rando, N., 2010, "A Review on Large Deployable Structures for Astrophysics Missions", Acta Astronautica, Vol. 67, No. 1-2, pp. 12-26. doi: 10.1016/j.actaastro.2010.02.021
Qi, Z.H., Xu, Y.S., Luo, X.M. and Yao, S.J., 2010, "Recursive Formulations for Multibody Systems with Frictional Joints Based on the Interaction Between Bodies", Multibody System Dynamics, Vol. 24, No. 2, pp. 133166. doi: 10.1007/s11044-010-9213-z

Rajaei, M. and Ahmadian, H., 2014, "Development of Generalized Iwan Model to Simulate Frictional Contacts with Variable Normal Loads", Applied Mathematical Modelling, Vol. 38, No. 15-16, pp. 4006-4018. doi: 10.1016/j.apm.2014.01.008

Sarkar, S., Venkatraman, K. and Dattaguru, B., 2004, "Dynamics of Flexible Structures with Nonlinear Joints", Journal of Vibration and Acoustics, Vol. 126. No. 1. pp. 92-100. doi: 10.1115/1.1596548

Shin, Y.S., and Lee, I., 2000, "Investigation of Equivalent System Modeling and Dynamic Characteristics Using Reduced Models", AIAA Journal, Vol. 38, No. 1, pp. 102-109. doi: 10.2514/2.928

Tian, Q., Zhang, Y.Q., Chen, L.P. and Flores, P., 2009, "Dynamics of Spatial Flexible Multibody Systems with Clearance and Lubricated Spherical Joints", Computers \& Structures, Vol. 87, No. 13-14, pp. 913-929. doi: 10.1016/j.compstruc.2009.03.006 